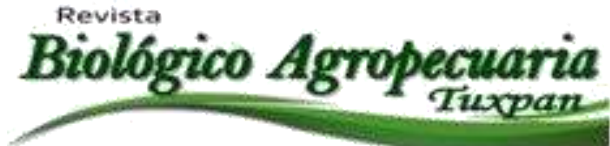

\title{
Aprovechamiento de las fibras de Agave angustifolia Haw, Usos alternativos
}

Use of Agave angustifolia Haw fiber, alternative uses

Hidalgo Reyes Martin ${ }^{凶}$, Magdaleno Caballero Caballero, Rodolfo Solano Gómez

Instituto Politécnico Nacional. Centro Interdisciplinario de Investigación para el Desarrollo Integral Regional, Unidad Oaxaca. Hornos No. 1003, Col. Noche Buena. Santa Cruz Xoxocotlán, Oaxaca.

México. *Universidad Autónoma Chapingo. km 38.5 Carr. México - Texcoco. CP 56230, Chapingo, Estado de México. Tel (595)952150.

${ }^{\bowtie}$ Autor para correspondencia: hogladi@hotmail.com

Recibido: 07/01/2014
Aceptado: 13/07/2014

\section{RESUMEN}

La milenaria historia cultural del territorio mexicano radica en su compleja variedad geográfica y biológica, esto incluye a la familia de las agavaceae, cuya diversidad cubre más de un centenar de especies con nombres y usos múltiples según la región, llegando a ser uno de los símbolos de identidad nacional. El presente trabajo analiza el uso integral del Agave angustifolia Haw, utilizado en la elaboración de la bebida alcohólica emblemática del estado de Oaxaca, el mezcal. Este agave, de gran importancia económica, constituye una fuente de empleo constante dentro de la región del Mezcal, representa la principal fuente de ingresos en forma directa o indirecta para más de 75000 familias; sin embargo, la planta no es aprovechada totalmente. La mayoría de los residuos del proceso son esparcidos en los campos agrícolas como desechos, algunos residuos son utilizados para elaborar compostas, pero no existe una aplicación específica. Las fibras tienen características ligno-celulósicas, lo cual representa un nicho de oportunidades. Los usos alternativos incluyen una amplia gama de productos. Se concluye que para el Agave angustifolia Haw no se han explorado todas las alternativas de uso posibles, se requiere innovar con este material y generar productos que marquen de manera significativa, el uso de los residuos.

Palabras claves: papel, celulosa, artesanías, compuestos.

\begin{abstract}
The ancient cultural history of Mexico lies in its complex geographical and biological variety, it includes the agavaceae family, whose diversity covers more than a hundred species with names and multiple uses by region, even becoming one of the symbols national identity. This paper analyzes the full use of Agave angustifolia Haw, chosen for its economic importance in the development of alcoholic beverage emblematic of the Oaxaca state, mezcal. This agave is a source of constant employment within the region of Mezcal, the main source of income directly or indirectly to more than
\end{abstract}


75000 families, but the plant is not fully exploited. Most process residues are scattered in agricultural fields as waste, some are used to make compost, but there is no particular specific application throughout the volume of waste. Alternative uses include a wide range of products, mainly handicraft. We conclude that for the Agave angustifolia Haw have not explored all possible alternatives, is required with this item innovate and create products that mark, significantly, the use of waste. The fibers of this plant ligno-cellulosic have characteristics similar to those of wood from the trees, this situation represents a niche opportunities for residual fibers in every way.

Keywords: paper, pulp, handicrafts, composites.

\section{INTRODUCCIÓN}

Linneo describió al género Agave en 1753. Del griego, el nombre del género significa "admirable". El área de distinción es muy amplia, se extiende desde Norteamérica hasta Colombia y Venezuela, reportándose 310 especies (Nóbel, 1998). En Oaxaca sehan localizado 23 especies, cultivados y silvestres, su uso principal es la fabricación de mezcal; se aprovechan alrededor de diez especies, pero destaca como preferido el Agave angustifolia Haw (Chagoya, 2004). Sin embargo, únicamente se emplea el tallo o piña. En otros países, los materiales con un contenido ligno celulósico son aprovechados industrialmente (Bessadok et al., 2008). Actualmente se prevé que las fibras lignocelulósicas derivadas de las plantas tengan una función importante en la transición respecto a las fibras sintéticas (FAO, 2004), pero aún no se tiene la caracterización de estos productos.

En Oaxaca existe un área territorial integrada por 146 localidades ubicadas en 9

Distritos de los Valles Centrales y parte de la Sierra Sur del Estado. En el $60 \%$ de estas localidades, las actividades económicas principales están ligadas al Agave, que constituye la base económica y fuente principal de trabajo e ingresos (Nobel, 1998).La industria del mezcal, después de la migración, es la segunda fuente de divisas para el Estado, además la simple reseña de los beneficios y usos del maguey es muy extensa. Existe una relación de usos históricos y actuales, de todo lo que puede hacerse con las distintas partes del agave: alimento, medicina, vestuario, vivienda, ornato, instrumentos de trabajo, diversión y cultura, e incluso como mortaja (BANCOMEXT, 1997).

El objetivo de este trabajo fue realizar un análisis del uso integral del Agave Angustifolia Haw, a partir del análisis de las condiciones específicas de producción de mezcal en el Estado de Oaxaca y determinar las posibles alternativas de uso para los residuos.

\section{MATERIALES Y MÉTODOS}

De manera general, el trabajo se desarrollo en tres fases: investigación documental, investigación de campo y finalmente el análisis y evaluación de datos. Los pasos a desarrollados en el presente trabajo fueronlosexpuestosacontinuación: identificación de una necesidad, planteamiento del problema y de la zona de estudio, búsqueda de información a través de una investigación documental o búsqueda bibliográfica, planteamiento de las variables de estudio, trabajo de campo y recolección de datos, análisis de datos, evaluación y obtención de resultados, finalmente la generación de conclusiones y recomendaciones.

\section{Revista Científica Biológico Agropecuaria Tuxpan 2 (1) ISSN: 2007-6940}




\section{RESULTADOS Y DISCUSIÓN}

En principio se expone brevemente el proceso de producción de mezcal, el cual se realiza en cinco niveles; autoconsumo, local, regional, nacional e internacional. La productividad es baja. Se obtienen 300 litros de mezcal en promedio, en un tiempo de 15 días y se requieren $3500 \mathrm{~kg}$ de agave. Las fases del proceso son (Silva, 1999):

Preparación de la materia prima. La cosecha se realiza en la madurez del agave ( 8 años aprox.), se cortan las hojas dejando limpio el tallo o "piña". Las piñas se seccionan y se transportan a las fábricas de mezcal.

Cocción de la piña del agave. Se realiza durante $72 \mathrm{~h}$, en un horno a flor de tierra de forma trunco-cónica revestido de piedras con capacidad de 4 a 6 toneladas.

$\square$ Seccionado y molienda. Para reducir las piñas en estado cocido se utilizan machetes y hachas. En la molienda se reduce más el tamaño del agave. Se utiliza un molino chileno.

$\square$ Fermentación. Se coloca el material en barricas de roble y se agrega del $5 \%$ al $10 \%$ de agua tibia. El tiempo de fermentación varía entre 72 y $200 \mathrm{~h}$.

$\square$ Destilación. Se efectúa en alambiques artesanales. Predominan los equipos rústicos, aunque hay algunos equipos industriales de destilación continua.

$\square$ Condensación-refinamiento. El proceso es netamente artesanal. La calidad del mezcal se determina de forma empírica en base a la experiencia del productor.

$\square$ Envasado. El envasado se realiza de forma manual y en casos muy raros de forma semiautomática.

El proceso de producción del mezcal es netamente artesanal, siendo una limitante en la productividad. Es importante considerar que toda la materia orgánica sobrante del proceso se convierte en un desecho que no genera un beneficio para el productor. Al inicio, todas las hojas que son cortadas en la cosecha son abandonadas en el campo y al final del proceso, el bagazo cocido también es considerado un desecho. Haciendo cálculos, la masa desperdiciada, está por arriba del $60 \%$ del material utilizado, dichos residuos contienen fibra lignocelulósica y de acuerdo a lo reportado por investigaciones previas, estos materiales pueden ser aprovechados de múltiples formas.

Con la fibra del agave es viable fabricar productos que brinden calidad, originalidad y distinción. Al mismo tiempo se promovería la responsabilidad ambiental y ayudaría a solidificar la raíz cultural a través de la creatividad e iniciativa. Con productos artesanales se ofrece presencia, prestigio $\mathrm{y}$ distinción en el mercado por el alto valor que representa la elaboración de estos productos. Como propuesta, algunos productos pueden ser: Aretes, cuadernos, cuadros, portarretratos, sobres, hojas de papel, agendas, llaveros, tarjetas, bolsas, entre otros.

Afortunadamente hace pocos años, se inició una etapa de desarrollo de tecnología para la región del Mezcal en Oaxaca. Instituciones como el Consejo Nacional de Ciencia y Tecnología (CONACYT), y el Centro Interdisciplinario de Investigación para el Desarrollo Integral Regional, unidad Oaxaca (CIIDIR - Oaxaca), han desarrollado proyectos para impulsar la generación de tecnologías adecuadas a los procesos productivos en la región.

\section{CONCLUSIONES}

Es necesario impulsar el desarrollo tecnológico en los procesos de producción para obtener un mayor aprovechamiento de los recursos disponibles. Actualmente en Oaxaca, 
Hidalgo et al., 2014

el único producto que se obtiene del Agave angustifolia Haw es el mezcal. Una gran cantidad de residuos, resultan de este proceso, y en su mayoría no son aprovechados. El bagazo cocido de agave y las vinazas no tienen un uso específico. Existen alternativas para aprovechar estos residuos, el más simple es hacer compostas y para reincorporar el material en los terrenos de cultivo. Los usos alternativos para las fibras de agave son muchos, entre otros esta la fabricación de productos artesanales, la elaboración de nuevos materiales compuestos con una gran gama de aplicaciones industriales, textiles y en la construcción.

\section{LITERATURA CITADA}

Banco Nacional de Comercio Exterior S.N.C. (BANCOMEXT). 1997. Mezcal: elixir de larga vida. Gobierno del Estado de Oaxaca. México. CVS Publicaciones. $123 \mathrm{p}$.

Bessadok, A., Marais, S., Roudesli, S., Lixon, C. y Metayor, M. 2008. Influence of chemical modifications on water sorption and mechanical properties of Agave fiber, Composites Part A, 39: 2945. https://doi.org/10.1016/j.compositesa.2007.09.007
Chagoya, M. V. M. 2004. Diagnóstico de la cadena productiva del sistema producto maguey mezcal. SAGARPA, SEDAF, COMMAC. Recuperado de http://www.oeidrusoaxaca.gob.mx/Estudios/diagnostico/SP MM\%20VERSION\%20FINAL.swf

F.A.O. (Food and Agriculture Organization). 2004. Los efectos ambientales de las fibras duras yel yute en aplicaciones industriales no textiles. Consulta fibras naturales.

http://www.fao.org/es/esc/common/ecg/ 343/es/esc_4_sp.pdf [Consulta 8.11.12].

Nóbel, P. S. 1998. Los incomparables agaves y cactus. Editorial Trillas. México. 211 Pp.

Silva, S. L. 1999, Tecnificación del proceso de fabricación del mezcal. Centro Interdisciplinario de Investigación para el Desarrollo Integral Regional (CIIDIR), OAXACA.

Copyright (c) 2014 Martin Hidalgo Reyes, Magdalena Caballero Caballero yR odolfo Solano Gómez

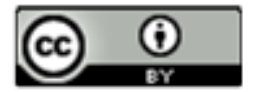

Este texto está proteg ido por una licencia Creative Commonz 4.0.

Usted es libre para Compartir —copiar y redistribuir el material en cualquier medio o formato- y Ad aptar el documento —remezclar, transformar y crear a partir del material- para cualquier propósito, inchso para fines comerciales, siempre que cumpla la condición de:

Atribución: Usted debe dar crédito a la obra original de manera adecuada, proporcionar un enlace a la licencia, e indicar si se han realizado cambios. Puede hacerlo en cualquier forma razonable, pero no de forma tal que sugiera que tiene el apoyo del licenciante olo recibe por el usoque hace de la obra.

Resumendelicencia - Textocompletodelalicenria

Revista Científica Biológico Agropecuaria Tuxpan 2 (1) ISSN: 2007-6940 